\title{
A KEPLER'S NOTE ON SECULAR INEQUALITIES
}

\author{
Nota del s.c. ANTONIO GIORGILLI (*)
}

(Adunanza del 5 maggio 2011)

SunTO. - In questa nota intendo illustrare il lavoro di Keplero sulle ineguaglianze secolari nei moti planetari esponendo il contenuto di un manoscritto pubblicato solo nel 1860. Nella sua memoria Keplero mette in evidenza la necessità di un modello che descriva le ineguaglianze secolari dei pianeti come variazioni periodiche rispetto ai valori medi. Il mio scopo è attirare l'attenzione su questo punto, che sembra essere stato sottovalutato. In effetti, i riferimenti al lavoro di Keplero si limitano tipicamente ad osservare che egli notò un rallentamento nel moto di Saturno ed un'accelerazione in quello di Giove.

ABSTRACT. - I discuss the problem of secular inequalities in Kepler by giving account of a manuscript note that has not been published until 1860. In his note Kepler points out the need for a model, clearly inspired by the method of epicycles, that describes the secular inequalities as periodic ones. I bring attention to this point, that seems to have been underestimated, since the references to Kepler's work usually report only that he observed a decreasing mean motion for Saturn and an increasing one for Jupiter.

\section{INTRODUCTION}

The discovery by Kepler of the elliptic shape of the planetery orbits is often considered as a discontinuity with the traditional models of Classical Astronomy, based on geometrical tools such as circles, epicycles

(*) Dipartimento di Matematica, Università degli Studi di Milano, via Saldini 50, 20133 Milano, Italy. E-mail: antonio.giorgilli@unimi.it 
and equants. The enthusiastic announcement of the discovery in chapter LVI of Astronomia Nova [6], where Kepler says that he was "quasi e somno expergefactus, et novam lucem intuitus" (like suddenly awakened from sleep, and seeing a new light) seems to confirm that this was his feeling, too. But, as often happens, reality turned out to be complex enough to escape our theories: the orbits of the planets are not exactly elliptic. This is well known today, but it seems that the circumstancy that long term deviations from the elliptic motion have been investigated in great detail by Kepler himself, with an explicit conjecture that these deviations should be periodic, is not so known, even among astronomers.

Kepler's opinion is clearly stated at the beginning of the preface to the Tabulz Rudolphinze [8], where he says: ${ }^{1}$

"Et de certitudine quidem calculi testabuntur observationes præsentium temporum, imprimis Braheanæ; de futuris vero temporibus plura præsumere non possumus, quam vel observationes veterum, quibus usus sum, vel ipsa motuum mediorum conditio, nondum penitus explorata, concursusque causarum physicarum prestare possunt, cum observationes Regiomontani et Waltheri testentur, omnino de æquationibus secularibus esse cogitandum, ut singulari libello reddam demonstratum suo tempore; quæ tamen æquationes quales et quantæ sint, ante plurimum sæculorum decursum observationesque eorum, a gente bumana definiri nequaquam possunt."

The booklet promised by Kepler was never published by him, and probably this caused the details about his work to be forgotten. A preliminary manuscript was found after his death, but it was not published until 1860, when it was included in the complete edition of Kepler's works [7]. The present memoir intends to give a short report on Kepler's note. ${ }^{2} \mathrm{~A}$ short account on further developments after Kepler is also included.

1 "And the observations made in our epoch, especially by Brahe, will prove the certainty of our calculations. However, concerning the future we can not expect so much. The validity may be questioned by ancient observations, that I'm well aware of, by the knowledge of the mean motions, that have not yet been fully explored, and by the concurrence of physical actions. The observations of Regiomontanus and Walther do indeed show that we should definitely think about secular equations, as I will explain in a specially devoted booklet. Which and how many equations we need, however, mankind will be unable to decide before many centuries of observations have been passed."

2 I should mention that my attention on Kepler's note was prompted by a 


\section{DATA FROM ANCIENT OBSERVATIONS}

As the title page of the Tabulx Rudolphinz says, the idea of compiling new astronomical tables was conceived by Tycho Brahe (15461601) since 1564 (he was 17years old), and the actual work was started after 1572. Kepler began his collaboration with Tycho Brahe in 1600, and after Brahe's death he undertook the job of continuing the compilation. He completed the tables by 1623 , but only on 1627 he could publish them. The observational data on which Kepler's work was based were largely due to his mentor Tycho Brahe, who had succeeded in measuring the positions of celestial bodies with a precision which he claimed to be 1' (actually about 2' in most cases), a great achievement for his epoch.

As it appears from the sentence quoted in the Introduction, Kepler was not just satisfied by the excellent agreement between the computational predictions based on his tables and Tycho Brahe's data. He had indeed undertaken a big work in order to compare his calculations with ancient observations available to him. This is the origin of his handwritten note.

Most of the ancient data available to Kepler were due to Johannes Müller der Könisberg (1436-1476), also named Regiomontanus, and Bernard Walther (1430-1504). They had collected a long series of observations between 1461 and 1504 which were considered as the most complete and precise at that time. Some data are due to Ptolemy (c.90-c.168), who also reports previous observations due to Chaldean astronomers (made around $229 \mathrm{BC}$ ) and to Dionysius (made around $270 \mathrm{BC}$ ). One observation is due to Copernicus.

Let me give a few examples of visual observations by Regiomontanus and Walther. This will illustrate how Kepler had to work in order to use them. The first example refers to Jupiter. ${ }^{3}$

"Anno 1478, 22 Aug. h. 3 post medium noctis fuerant in una linea 4 et duo oculi $\succ$, et erat 4 occidentalior, distans per medietatem distantiz, qua duo oculi distant, ab oculo occidentaliori; sic visui apparuit."

conference given by J. Laskar at the Istituto Lombardo Accademia di Scienze e Lettere[20]. The present note can be considered as complementary to Laskar's memoir.

3 "In 1478, august 22, tree hours past midnight, Jupiter and the two eyes of Taurus were on the same line, and Jupiter was toward west, the distance from the west eye of Taurus being half the distance between the two eyes; so it appeared to the eye." 
Tab. 1 - Observed positions of Saturn compared with the calculated ones.

The first column reports the date of the observation; the second one the longitude of the observed planet as reconstructed by Kepler on the basis of the observational data

by Regiomontanus and Walther; the third one the longitude predicted by Kepler; the fourth one the difference. The word "idem" in the first column means that Kepler gives two possible outcomes for his determinations of the longitude of the planet.

\begin{tabular}{|c|c|c|c|c|c|}
\hline Date & Observed & & Calculated & & Difference \\
\hline 2.12 .1461 & $29^{\circ} 38^{\prime}$ & 6 & $29^{\circ} 14^{\prime} \frac{1}{2}$ & 6 & $-23^{\prime} \frac{1}{2}$ \\
\hline idem & $29^{\circ} 5^{0^{\prime}}$ & 6 & $29^{\circ} 14^{\prime} \frac{1}{2}$ & 6 & $-35^{\prime} \frac{1}{2}$ \\
\hline 17.09 .1475 & $5^{\circ} 3^{8^{\prime} \frac{1}{2}}$ & $\Omega$ & $4^{\circ} 42^{\prime}$ & $\Omega$ & $-56^{\prime} \frac{1}{2}$ \\
\hline idem & $5^{\circ} 54^{\prime}$ & $\Omega$ & $4^{\circ} 42^{\prime}$ & $\delta$ & $-1^{\circ} 12^{\prime}$ \\
\hline 25.03 .1476 & $0^{\circ} 49^{\prime}$ & $\Omega$ & $0^{\circ} 6^{\prime}$ & $\Omega$ & $-43^{\prime}$ \\
\hline 13.10 .1476 & $21^{\circ} 32^{\prime}$ & $\Omega$ & $20^{\circ} 46^{\prime} \frac{1}{2}$ & $\Omega$ & $-45^{\prime} \frac{1}{2}$ \\
\hline 9.10 .1477 & $2^{\circ} 13^{\prime}$ & $m$ & $1^{\circ} 23^{\prime}$ & $m$ & $-50^{\prime}$ \\
\hline idem & $2^{\circ} 28^{\prime}$ & $m$ & $1^{\circ} 23^{\prime}$ & mp & $-1^{\circ} 5^{\prime}$ \\
\hline 19.04 .1478 & $28^{\circ} 10^{\prime}$ & $\delta$ & $27^{\circ} 18^{\prime}$ & $\delta$ & $-52^{\prime}$ \\
\hline idem & $28^{\circ} 36^{\prime}$ & $\Omega$ & $27^{\circ} 18^{\prime}$ & $\Omega$ & $-1^{\circ} 18^{\prime}$ \\
\hline 24.09.1478 & $12^{\circ} 15^{\prime}$ & mo & $11^{\circ} 32^{\prime}$ & mp & $-43^{\prime}$ \\
\hline 30.10 .1479 & $27^{\circ} 28^{\prime} \frac{1}{2}$ & $m$ & $26^{\circ} 5^{0^{\prime}}$ & mp & $-38^{\prime} \frac{1}{2}$ \\
\hline 22.10 .1481 & $21^{\circ} 13^{\prime}$ & $\Omega$ & $20^{\circ} 45^{\prime} \frac{1}{2}$ & $\Omega$ & $-27^{\prime} \frac{1}{2}$ \\
\hline idem & $21^{\circ} 25^{\prime}$ & $\Omega$ & $20^{\circ} 45^{\prime} \frac{1}{2}$ & $\Omega$ & $-39^{\prime} \frac{1}{2}$ \\
\hline 12.01 .1482 & $25^{\circ} 11^{\prime}$ & $\Omega$ & $24^{\circ} 31^{\prime}$ & $\Omega$ & $-40^{\prime}$ \\
\hline 21.11 .1484 & $23^{\circ} 30^{\prime}$ & $m$ & $22^{\circ} 5^{0^{\prime}}$ & $m$ & $-4 \mathrm{o}^{\prime}$ \\
\hline 15.10 .1503 & $17^{\circ} 46^{\prime} \frac{1}{2}$ & 6 & $17^{\circ} \mathrm{o}^{\prime} \frac{1}{2}$ & 6 & $-46^{\prime}$ \\
\hline idem & $17^{\circ} 58^{\prime}$ & 9 & $17^{\circ} \mathrm{o}^{\prime} \frac{1}{2}$ & 6 & $-58^{\prime} \frac{1}{2}$ \\
\hline 11.12 .1503 & $15^{\circ} 30^{\prime}$ & 6 & $14^{\circ} 51^{\prime} \frac{1}{2}$ & 6 & $-38^{\prime} \frac{1}{2}$ \\
\hline idem & $15^{\circ} 34^{\prime} \frac{1}{2}$ & 6 & $14^{\circ} 51^{\prime} \frac{1}{2}$ & 6 & $-43^{\prime}$ \\
\hline 8.02 .1504 & $11^{\circ} 11^{\prime}$ & ซ & $10^{\circ} 29^{\prime}$ & ๑ & $-42^{\prime}$ \\
\hline idem & $11^{\circ} 15^{\prime} \frac{1}{2}$ & 6 & $10^{\circ} 29^{\prime}$ & 6 & $-46^{\prime} \frac{1}{2}$ \\
\hline 8.03 .1504 & $10^{\circ} 5^{1^{\prime}}$ & 6 & $10^{\circ} 5^{\prime}$ & 6 & $-46^{\prime}$ \\
\hline 29.03 .1504 & $11^{\circ} 34^{\prime}$ & 6 & $10^{\circ} 46^{\prime}$ & ๑ & $-48^{\prime}$ \\
\hline 24.05 .1504 & $16^{\circ} 19^{\prime}$ & ช & $15^{\circ} 37^{\prime} \frac{1}{2}$ & כ) & $-41^{\prime} \frac{1}{2}$ \\
\hline 24.02 .1514 & $25^{\circ} 13^{\prime} \frac{1}{3}$ & $m$ & $24^{\circ} 56^{\prime} \frac{2}{3}$ & $m$ & $-16^{\prime} \frac{1}{3}$ \\
\hline
\end{tabular}


Tab. 2 - Observed positions of Jupiter compared with the calculated ones.

\begin{tabular}{|c|c|c|c|c|c|}
\hline Date & Observed & & Calculated & & Difference \\
\hline 20.03 .1462 & $2^{\circ} 15^{\prime}$ & 6 & $2^{\circ} 21^{\prime} \frac{3}{4}$ & 6 & $+6^{\prime} \frac{3}{4}$ \\
\hline 26.04 .1468 & $29^{\circ} 24^{\prime} \frac{2}{3}$ & II & $29^{\circ} 24^{\prime} \frac{2}{3}$ & II & $\mathrm{o}^{\prime}$ \\
\hline 29.04 .1468 & $29^{\circ} 56^{\prime} \frac{1}{2}$ & II & $29^{\circ} 56^{\prime} \frac{1}{2}$ & II & $\mathrm{o}^{\prime}$ \\
\hline 15.03 .1471 & $29^{\circ} 33^{\prime}$ & mp & $29^{\circ} 16^{\prime}$ & mp & $+17^{\prime}$ \\
\hline 21.02 .1478 & $24^{\circ} 33^{\prime}$ & $\Upsilon$ & $25^{\circ} \mathrm{o}^{\prime}$ & $\Upsilon$ & $+27^{\prime}$ \\
\hline 22.08 .1478 & $0^{\circ} 25^{\prime} \frac{1}{2}$ & II & $0^{\circ} 39^{\prime} \frac{1}{2}$ & II & $+14^{\prime}$ \\
\hline 30.09 .1478 & $0^{\circ} 24^{\prime}$ & II & $0^{\circ} 42^{\prime} \frac{1}{2}$ & II & $+18^{\prime} \frac{1}{2}$ \\
\hline 21.11.1484 & $23^{\circ} 33^{\prime}$ & II & $23^{\circ} 4^{\prime} \frac{1}{2}$ & II & $+15^{\prime}$ \\
\hline 10.01 .1488 & $23^{\circ} 51^{\prime} \frac{1}{2}$ & $\approx$ & $24^{\circ} 2^{\prime}$ & $\approx$ & $+10^{\prime} \frac{1}{2}$ \\
\hline 15.02 .1497 & $12^{\circ} 3^{8^{\prime} \frac{3}{4}}$ & $x^{7}$ & $12^{\circ} 38^{\prime} \frac{3}{4}$ & $x^{\top}$ & $\mathrm{o}^{\prime}$ \\
\hline 8.09 .1503 & $11^{\circ} 37^{\prime}$ & б & $11^{\circ} 5^{2^{\prime}}$ & ซ & $+18^{\prime}$ \\
\hline 19.01 .1504 & $6^{\circ} 31^{\prime} \frac{2}{3}$ & 6 & $6^{\circ} 5^{0^{\prime}}$ & ช & $+18^{\prime} \frac{1}{3}$ \\
\hline 9.02 .1504 & $4^{\circ} 57^{\prime} \frac{1}{2}$ & 6 & $5^{\circ} 22^{\prime} \frac{1}{3}$ & 6 & $+24^{\prime} \frac{5}{6}$ \\
\hline 29.04.1504 & $11^{\circ} 37^{\prime}$ & 6 & $11^{\circ} 5^{2^{\prime}}$ & б & $+15^{\prime}$ \\
\hline 24.05 .1504 & $16^{\circ} 11^{\prime} \frac{1}{2}$ & 6 & $16^{\circ} 35^{\prime}$ & 6 & $+23^{\prime} \frac{1}{2}$ \\
\hline
\end{tabular}

It should be remarked that this is an example of a quite precise observation, since the position of Jupiter is given with reference to two well identified fixed stars that can be found in a good catalog. In other cases only the relative position of two or more moving objects is given, typically conjunctions among two or more planets and/or the Moon. ${ }^{4}$

"1478, 24 Sept, 40” ante ortum solis vidi lunam circa $\hbar$, quasi coniunctos; distabat Luna modicum ad septentrionem, ita ut inter circunferentiam eius et $\hbar$ videretur mediare spatium unius palmx."

In this case Kepler determines the longitude of the Moon, which

4 "In 1478, September 24, 40 minutes before sunrise I saw Saturn and the Moon approximately in conjunction; Moon's position was scanty on the north direction, and it appeared that between her circle and Saturn one could insert the width of a palm." 
allows him to evaluate the longitude of Saturn. Sometimes the indications of Regiomontanus are definitely more challenging.'

“(...) stella 4 videbatur inter duas Virginis, quarum lucidior est circa medietatem ale sinistre Virginis, alia obscurior circa oculum eius versus Leonem (...)"

Kepler's comment is: "Magna cum perplexitate diu conflictatus sum, qænam essent bæc duæ stellæ" (With great perplexity and for a long I racked my brain trying to figure out which these two stars are). Then he makes an hypothesis that seems reasonable, and proceeds with the calculation.

After 1488 Walther began to observe with the help or zodiacal armillary spheres, so he could give longitudes and latitudes in degrees and minutes of arc. He was also used to add notes concerning the reliability of his observations. ${ }^{6}$

Kepler actually made a major effort in reconstructing the reported observations so as to find the longitudes and often also the latitudes of the planets, which allowed him to make a comparison with the tables. He also devotes some pages to a discussion of the reliability of the measured data, possibly affected by a bad setting of the armillary spheres, or by incorrect time indications. Sometimes he points out the possible existence of typographical errors. I will not discuss this aspect here.

A synopsis of Kepler's results is reported in Tabs. 1-5. I collected only data for the longitudes of the planets. ${ }^{7}$ In many cases Kepler determines also the latitude, but these data appear not to be very relevant for the discussion concerning secular terms, so I omit them.

5 "(...) the star Jupiter was seen between two stars of Virgo. The brighter one is close to the center of the left wing of Virgo, the other one, less bright, is close to her eye, towards Leo (...)"

6 The report of Walther's observations has been published by Johann Schöner in 1544 (see [22]). A rather detailed account of the data collected by Walther in 1503. 1504 can be found in [10] and [11].

7 In Kepler's notations, the circle of the ecliptic is divided into 12 sectors of $30^{\circ}$ each, identified by the constellations, and the longitude is given relative to the constellation. E.g., the notation $17^{\circ} 13^{\prime} \bumpeq$ means the longitude $17^{\circ} 13^{\prime}$ measured from the west point of the constellation of Libra. Following Kepler, in the text and in the tables I use the common astronomical symbols for the constellations. For the reader's convenience I include here a list: $\uparrow$ (Aries), $\succ$ (Taurus), II (Gemini), 6 (Cancer), $\Omega$ (Leo), mp (Virgo), $\Omega$ (Libra), m (Scorpio), × (Sagittarius), 6 (Capricornus), $\approx$ (Aquarius), $\boldsymbol{x}$ (Pisces). 


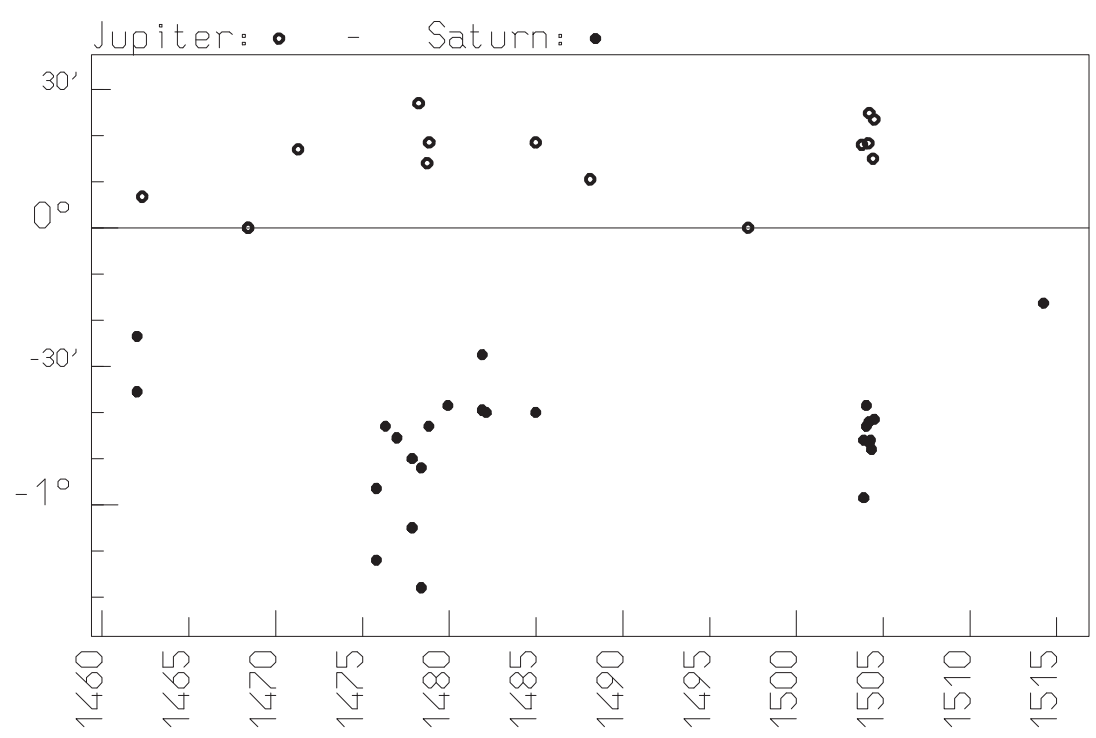

Fig. 1 -Difference between the longitudes of Jupiter and Saturn calculated by Kepler from the Tabulæ Rudolphinæ and the positions evaluated from the observations of Regiomontanus and Walther, vs. the observation date. The plotted values come from the last column of Tabs. 1 and 2. The graph clearly shows that Jupiter seems to move faster than predicted, while Saturn is slower.

A plot of the last column in the tables, namely the differences between the calculated positions and the observed ones, is reported in Fig. 1 for Jupiter and Saturn and in Fig. 2 for Mercury, Venus and Mars. The observational errors are quite big, which is not surprising if one recalls the few examples that I have given; thus the data point have a significant dispersion. However, the first figure clearly exhibits a systematic deviation for Jupiter ad Saturn: the difference for Jupiter is always positive (about +18 ' according to Kepler), while for Saturn it is always negative (about $\left.-43^{\prime}\right)$. That is, Jupiter moves faster than predicted, while Saturn is slower. No systematic deviation occurs instead for the three smaller planets, although Kepler's claims that some exists also for Mars.

\section{THE NEED FOR SECULAR EQUATIONS}

From Kepler's considerations it seems that he concluded for the 
necessity of secular equations ${ }^{8}$ after a very careful examination of the last observation of a great conjunction between Jupiter and Saturn, by Walther. Walther's notes report that he observed the conjunction in the evening of may 24, 1504 (he died on June 19). However, according to Kepler's calculations the distance between the two planets should have been about $1^{\circ}$ at that time. Kepler writes:?

"Et hic dissensus calculi in 4 and $\hbar$, excurrens ad integrum gradum, est remora illa, quæ me, plurima perplexitate circumventum, per solidos quinque menses in observationibus Waltherianis exercuit tandemque ad nova consilia circa motuum mediorum speculationem adegit, deprehensa manifesta inequalitate motuum seculari. (Absolvi bucusque 18 Junii 1624.)"

Having thus decided that a secular equation is needed Kepler turns to determining it. Here he faces big difficulties, partly due to the lack of data, partly to an hypothesis that he strongly wants to be satisfied (although this may even appear as foolish to us).

Let me discuss this part in some detail: this will enlight which secular equation he was looking for.

Kepler starts by examining five ancient data for Saturn reported by Ptolemy. The first one refers to an observation made by Chaldean astronomers: ${ }^{10}$ "... a. 82 die 2 Xantichi vesperi, quod ex fide Ptolemxi interpretis fuit ante Chr. anno 229 d. 1 Mart. Tunc $\hbar$ sub australi bumero mp visus est 2 digitos". Four more observations are due to Ptolemy himself. Three of them are $\dot{\alpha} \kappa \rho о v v \chi 0 \mathrm{c}$ (i.e., with no time indication) and give the calculated oppositions of Saturn with the average Sun that occurred in $1^{\circ} 13^{\prime} \bumpeq, 9^{\circ} 40^{\prime} x^{\prime}$ and $14^{\circ} 14^{\prime} \sigma$. The fourth one reports

8 It should be stressed here that the meaning assigned by Kepler to the word "equation" corresponds rather to what we call "function". In fact he was looking for a (periodic) correction of the mean motions with respect to a fixed mean value.

9 "And this discrepancy in the calculation for Jupiter and Saturn, which amounts to a whole degree, is such an obstacle that it caused me to be assailed by many perplexities, and for five solid months I have been troubled until I eventually came to think about average motions in a different way, having accepted the manifest secular inequality of the motions. (I came to this conclusion on June 18, 1624.)"

10 " $\ldots$ in the evening of the second day of the Xantic month of the year 82, which according to the interpreters of Ptolemy is March 1, 229 BC. Then Saturn was seen two fingers below the austral shoulder of Virgo". 
Tab. 3 - Observed positions of Mars compared with the calculated ones.

\begin{tabular}{|c|c|c|c|c|c|}
\hline Date & \multicolumn{2}{|l|}{ Observed } & \multicolumn{2}{|l|}{ Calculated } & Difference \\
\hline 2.12 .1461 & $27^{\circ} 5^{\prime} \frac{1}{2}$ & 6 & $28^{\circ} 4^{\prime}$ & 6 & $+58^{\prime} \frac{1}{2}$ \\
\hline 24.12 .1461 & $14^{\circ} 17^{\prime}$ & $\approx$ & $15^{\circ} 23^{\prime}$ & $\approx$ & $+1^{\circ} 6^{\prime}$ \\
\hline 15.09 .1462 & $21^{\circ} 57^{\prime} \frac{1}{2}$ & $\Omega$ & $22^{\circ} 1^{\prime}$ & $\Omega$ & $+3^{\prime} \frac{1}{2}$ \\
\hline 4.10 .1462 & $10^{\circ} 56^{\prime}$ & $m$ & $10^{\circ} 39^{\prime}$ & $m$ & $-17^{\prime}$ \\
\hline 11.12 .1464 & $28^{\circ} 42^{\prime} \frac{1}{2}$ & $\Omega$ & $28^{\circ} 29^{\prime}$ & $\Omega$ & $-13^{\prime} \frac{1}{2}$ \\
\hline 19.06 .1465 & $1^{\circ} 57^{\prime} \frac{1}{2}$ & 6 & $2^{\circ} 13^{\prime}$ & 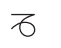 & $+15^{\prime} \frac{1}{2}$ \\
\hline 26.04 .1468 & $29^{\circ} 24^{\prime} \frac{1}{2}$ & II & $29^{\circ} 24^{\prime} \frac{1}{2}$ & II & $\mathrm{o}^{\prime}$ \\
\hline 26.07.1471 & $0^{\circ} 22^{\prime}$ & II & $0^{\circ} 31^{\prime} \frac{1}{2}$ & II & $+9^{\prime} \frac{1}{2}$ \\
\hline 7.03 .1474 & $0^{\circ} 42^{\prime}$ & $\Omega$ & $1^{\circ} 19^{\prime}$ & $\Omega$ & $+37^{\prime}$ \\
\hline 18.09 .1475 & $5^{\circ} 54^{\prime}$ & $\Omega$ & $5^{\circ} 38^{\prime} \frac{1}{2}$ & $\Omega$ & $-15^{\prime} \frac{1}{2}$ \\
\hline 24.01.1476 & $21^{\circ} 44^{\prime}$ & $m$ & $22^{\circ} 36^{\prime}$ & $m p$ & $-51^{\prime} \frac{1}{2}$ \\
\hline 19.02 .1476 & $16^{\circ} 53^{\prime}$ & $m$ & $16^{\circ} 41^{\prime}$ & $m$ & $-18^{\prime}$ \\
\hline 12.04 .1476 & $5^{\circ} 7^{\prime} \frac{1}{3}$ & $m$ & $4^{\circ} 55^{\prime} \frac{1}{2}$ & mp & $-11^{\prime} \frac{5}{6}$ \\
\hline 15.10 .1477 & $5^{\circ} 49^{\prime}$ & $m$ & $5^{\circ} 24^{\prime} \frac{1}{3}$ & $m$ & $-24^{\prime} \frac{2}{3}$ \\
\hline 16.03 .1478 & $27^{\circ} 6^{\prime} \frac{1}{2}$ & $\Omega$ & $27^{\circ} 23^{\prime}$ & $\Omega$ & $+16^{\prime} \frac{1}{2}$ \\
\hline 19.05.1478 & $12^{\circ} 7^{\prime} \frac{1}{2}$ & $\Omega$ & $12^{\circ} 15^{\prime}$ & $\Omega$ & $-3^{\prime} \frac{1}{2}$ \\
\hline
\end{tabular}

(To be continued).

that that Saturn was observed" ${ }^{11}$ in $9^{\circ} 15^{\prime} \approx$, quando $D$ est observata dimidio gradus ultra, i.e. in $9^{\circ} 45^{\prime} \approx$; observatus est $\hbar$ ad claram Hyadum, sed lubricze sunt circumstantix".

First Kepler recalculates the longitudes of the oppositions with the true position of the Sun, also taking into account the renumbering of zodiacal signs with respect to Ptolemy's time, which according to Kepler corresponds to $1^{\circ} 3^{\prime}$. Adding both corrections he finds $2^{\circ} 28^{\prime}, 10^{\circ} 43^{\prime} x^{\prime}$ and $15^{\circ} 10^{\prime} \sigma$. Then he keeps fixed the eccentricity of Saturn's orbit,

11 “at $9^{\circ} 15^{\prime} \approx$, when the Moon was seen half a degree forward, i.e., at $9^{\circ} 45^{\prime}$; Saturn was observed with respect to the star named 'clara hyadum', but the circumstances are fallacious." (The star 'clara hyadum' is Aldebaran.) 
Tab. 3 -(Continued).

\begin{tabular}{|c|c|c|c|c|c|}
\hline Date & Observed & & Calculated & & Difference \\
\hline 30.10 .1479 & $27^{\circ} 24^{\prime}$ & $m$ & $27^{\circ} 28^{\prime} \frac{1}{2}$ & $m$ & $+4^{\prime} \frac{1}{2}$ \\
\hline 15.11 .1479 & $20^{\circ} 56^{\prime}$ & $\Omega$ & $21^{\circ} 7^{\prime} \frac{1}{2}$ & $\Omega$ & $+11^{\prime} \frac{1}{2}$ \\
\hline 28.11 .1484 & $25^{\circ} 52^{\prime}$ & $\Upsilon$ & $25^{\circ} 34^{\prime} \frac{1}{2}$ & $\Upsilon$ & $-18^{\prime} \frac{1}{2}$ \\
\hline 20.08 .1486 & $8^{\circ} \mathrm{o}^{\prime}$ & $\Upsilon$ & $8^{\circ} 4^{\prime}$ & $\Upsilon$ & $+4^{\prime}$ \\
\hline 7.09 .1486 & $17^{\circ} 8^{\prime}$ & II & $17^{\circ} 14^{\prime} \frac{2}{3}$ & II & $+6^{\prime} \frac{2}{3}$ \\
\hline 13.09 .1488 & $11^{\circ} 21^{\prime}$ & ซ & $11^{\circ} 21^{\prime} \frac{1}{2}$ & 6 & $+\mathrm{o}^{\prime} \frac{1}{2}$ \\
\hline 3.10 .1488 & $21^{\circ} 12^{\prime} \frac{1}{2}$ & 6 & $21^{\circ} 39^{\prime} \frac{1}{3}$ & ซ & $+26^{\prime} \frac{5}{6}$ \\
\hline 18.09 .1490 & $0^{\circ} 12^{\prime} \frac{1}{2}$ & ซ & $0^{\circ} 15^{\prime} \frac{1}{2}$ & 6 & $+3^{\prime}$ \\
\hline 23.03.1491 & $22^{\circ} 37^{\prime}$ & $\Omega$ & $22^{\circ} 31^{\prime}$ & $\Omega$ & $-6^{\prime}$ \\
\hline 26.09 .1492 & $19^{\circ} 9^{\prime} \frac{1}{2}$ & $\Omega$ & $19^{\circ} 13^{\prime} \frac{1}{2}$ & $\Omega$ & $+6^{\prime}$ \\
\hline 27.03.1493 & $3^{\circ} 2^{\prime} \frac{1}{2}$ & $\Omega$ & $3^{\circ} 11^{\prime}$ & $\Omega$ & $+8^{\prime} \frac{1}{2}$ \\
\hline 30.09 .1503 & $11^{\circ} 33^{\prime} \frac{1}{3}$ & 6 & $11^{\circ} 57^{\prime}$ & 9 & $+23^{\prime} \frac{2}{3}$ \\
\hline 6.10 .1503 & $14^{\circ} 23^{\prime} \frac{1}{2}$ & 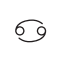 & $14^{\circ} 34^{\prime} \frac{1}{2}$ & 6 & $+11^{\prime} \frac{1}{2}$ \\
\hline 16.11 .1503 & $23^{\circ} 30^{\prime}$ & 6 & $23^{\circ} 59^{\prime}$ & 6 & $+29^{\prime}$ \\
\hline 19.01 .1504 & $6^{\circ} 31^{\prime} \frac{1}{2}$ & 6 & $6^{\circ} 17^{\prime} \frac{2}{3}$ & 6 & $-13^{\prime} \frac{5}{6}$ \\
\hline 6.02 .1504 & $4^{\circ} 57^{\prime} \frac{1}{2}$ & ซ & $4^{\circ} 56^{\prime}$ & 6 & $-1^{\prime} \frac{1}{2}$ \\
\hline 3.03 .1504 & $10^{\circ} 57^{\prime}$ & ซ & $11^{\circ} 10^{\prime}$ & ซ & $+13^{\prime}$ \\
\hline 30.05 .1504 & $20^{\circ} 4^{\prime} \frac{1}{2}$ & $\Omega$ & $20^{\circ} 11^{\prime} \frac{1}{2}$ & $\Omega$ & $+7^{\prime} \frac{1}{2}$ \\
\hline
\end{tabular}

"quæ bodie ex accuratissimis observationibus 30 continuorum annorum stabilitur" (which today is well established in view of 30 continuous years of very accurate observations), and by trying different positions of the aphelion he calculates the mean motion that gives him the best approximation of Ptolemy's longitudes. Here, he clearly assumes that the Earth's motion is uniform over all centuries, and calculates the intervals between two oppositions by Saturn's motion on the ellipse. This represents a first partial success, since it allows him to fit the observations within 10'. After that he comes to fit also the fourth observation. He can determine quite accurately the longitude and the time thanks to the position of the Moon, which is reported by Ptolemy. 
Tab. 4 - Observed positions of Venus compared with the calculated ones.

\begin{tabular}{|c|c|c|c|c|c|}
\hline Date & Observed & & Calculated & & Difference \\
\hline 14.12 .1461 & $0^{\circ} 7^{\prime}$ & $\approx$ & $0^{\circ} 44^{\prime}$ & $\approx$ & $+37^{\prime}$ \\
\hline idem & $0^{\circ} 31^{\prime}$ & $\approx$ & $0^{\circ} 44^{\prime}$ & $\approx$ & $+13^{\prime}$ \\
\hline 10.01 .1462 & $4^{\circ} 52^{\prime}$ & $x$ & $4^{\circ} 3^{\prime}$ & $x$ & $-49^{\prime}$ \\
\hline idem & $4^{\circ} 5^{\prime}$ & $x$ & $4^{\circ} 3^{\prime}$ & $x$ & $-2^{\prime}$ \\
\hline idem & $3^{\circ} 22^{\prime}$ & $x$ & $4^{\circ} 3^{\prime}$ & $x$ & $+41^{\prime}$ \\
\hline 19.09 .1462 & $20^{\circ} 16^{\prime}$ & $\Omega$ & $20^{\circ} 44^{\prime}$ & $\Omega$ & $+28^{\prime}$ \\
\hline 26.09 .1462 & $28^{\circ} 42^{\prime}$ & $\Omega$ & $28^{\circ} 35^{\prime}$ & $\Omega$ & $-6^{\prime}$ \\
\hline 20.10 .1462 & $27^{\circ} 37^{\prime}$ & mp & $26^{\circ} 45^{\prime} \frac{1}{2}$ & $m$ & $+8^{\prime} \frac{1}{2}$ \\
\hline 25.10 .1462 & $2^{\circ} 38^{\prime}$ & $\Omega$ & $2^{\circ} 47^{\prime}$ & $\Omega$ & $+9^{\prime}$ \\
\hline 21.02 .1478 & $24^{\circ} 5 \mathrm{o}^{\prime}$ & $\Upsilon$ & $25^{\circ} \mathrm{o}^{\prime}$ & $\Upsilon$ & $+10^{\prime}$ \\
\hline 11.08 .1478 & $10^{\circ} 51^{\prime} \frac{1}{2}$ & 6 & $10^{\circ} 48^{\prime}$ & 6 & $-3^{\prime} \frac{1}{2}$ \\
\hline 15.11 .1481 & $16^{\circ} 54^{\prime}$ & $\Omega$ & $16^{\circ} 29^{\prime}$ & $\Omega$ & $-25^{\prime}$ \\
\hline idem & $16^{\circ} 46^{\prime}$ & $\Omega$ & $16^{\circ} 29^{\prime}$ & $\Omega$ & $-17^{\prime}$ \\
\hline 19.11.1481 & $21^{\circ} 22^{\prime}$ & $\Omega$ & $20^{\circ} 57^{\prime} \frac{1}{2}$ & $\Omega$ & $-24^{\prime} \frac{1}{2}$ \\
\hline 25.11 .1481 & $27^{\circ} 52^{\prime}$ & $\Omega$ & $28^{\circ} \mathrm{o}^{\prime}$ & $\Omega$ & $-3^{\prime}$ \\
\hline idem & $28^{\circ} 14^{\prime}$ & $\Omega$ & $28^{\circ} \mathrm{o}^{\prime}$ & $\Omega$ & $+14^{\prime}$ \\
\hline 20.09 .1486 & $23^{\circ} 6^{\prime}$ & $\delta$ & $23^{\circ} 4^{\prime}$ & $\Omega$ & $-2^{\prime}$ \\
\hline 24.09 .1486 & $27^{\circ} 30^{\prime} \frac{3}{4}$ & $\Omega$ & $27^{\circ} 54^{\prime}$ & $\Omega$ & $+24^{\prime} \frac{3}{4}$ \\
\hline
\end{tabular}

(To be continued).

Finally, he tries to fit also the Chaldean observation, and he succeeds again, but with a further minor change of the mean motion. Here are Kepler's results for the five observations; the first line gives the predictions, the second one the data recalculated by Kepler by including the corrections mentioned above. For the fourth observation of Ptolemy he gives two possible corrections, the second one based on Moon's position.

$$
\begin{aligned}
& 1^{\circ} 36^{\prime} \bumpeq, 10^{\circ} 38^{\prime} x^{\prime}, 15^{\circ} 10^{\prime} \text { б, } 10^{\circ} 22^{\prime} \approx, 8^{\circ} 56^{\prime} 23^{\prime \prime} \mathrm{mp} \text {, pro } \\
& 2^{\circ} 16^{\prime} \bumpeq, 10^{\circ} 43^{\prime} x^{\prime}, 15^{\circ} 17^{\prime} \sigma, 10^{\circ} 18^{\prime} \approx, 8^{\circ} 41^{\prime} 0 \text { " mp, } \\
& \text { vel } 10^{\circ} 27^{\prime} \text { ex D. }
\end{aligned}
$$


Tab. 4 - (Continued).

\begin{tabular}{|c|c|c|c|c|c|}
\hline Date & \multicolumn{2}{|l|}{ Observed } & \multicolumn{2}{|l|}{ Calculated } & Difference \\
\hline 1.04 .1489 & $27^{\circ} 54^{\prime}$ & 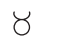 & $28^{\circ} 6^{\prime}$ & $\zeta$ & $+12^{\prime}$ \\
\hline 13.12 .1490 & $15^{\circ} 45^{\prime}$ & $\approx$ & $15^{\circ} 5 \mathrm{o}^{\prime}$ & $\approx$ & $+5^{\prime}$ \\
\hline 17.01.1491 & $23^{\circ} 26^{\prime}$ & $\approx$ & $23^{\circ} 35^{\prime} \frac{1}{2}$ & $\approx$ & $+9^{\prime} \frac{1}{2}$ \\
\hline 14.02 .1491 & $15^{\circ} 34^{\prime}$ & $\Upsilon$ & $16^{\circ} 10^{\prime}$ & $\Upsilon$ & $+36^{\prime}$ \\
\hline 19.09 .1494 & $22^{\circ} 30^{\prime}$ & $\delta$ & $22^{\circ} 27^{\prime} \frac{1}{2}$ & $\delta$ & $-2^{\prime} \frac{1}{2}$ \\
\hline 10.12 .1503 & $2^{\circ} 4^{\prime} \frac{1}{2}$ & $\approx$ & $2^{\circ} 3^{\prime}$ & $\approx$ & $-1^{\prime} \frac{1}{2}$ \\
\hline 19.01 .1504 & $17^{\circ} 36^{\prime} \frac{1}{2}$ & 6 & $18^{\circ} 12^{\prime}$ & 6 & $+35^{\prime} \frac{1}{2}$ \\
\hline 24.01 .1504 & $16^{\circ} 51^{\prime} \frac{1}{2}$ & б & $17^{\circ} 6^{\prime}$ & 7 & $+14^{\prime} \frac{1}{2}$ \\
\hline 27.01 .1504 & $16^{\circ} 41^{\prime} \frac{1}{2}$ & б & $17^{\circ} 4^{\prime}$ & 7 & $+22^{\prime} \frac{1}{2}$ \\
\hline 20.02 .1504 & $26^{\circ} 40^{\prime}$ & 7 & $27^{\circ} 1^{\prime}$ & б & $+21^{\prime}$ \\
\hline 3.03 .1504 & $6^{\circ} 26^{\prime}$ & $\approx$ & $6^{\circ} 39^{\prime}$ & $\approx$ & $+13^{\prime}$ \\
\hline 11.03 .1504 & $13^{\circ} 5^{8^{\prime}}$ & $\approx$ & $13^{\circ} 54^{\prime}$ & $\approx$ & $-4^{\prime}$ \\
\hline 12.03 .1504 & $15^{\circ} \mathrm{o}^{\prime}$ & $\approx$ & $14^{\circ} 50^{\prime}$ & $\approx$ & $-10^{\prime}$ \\
\hline 17.03 .1504 & $19^{\circ} 46^{\prime} \frac{1}{2}$ & $\approx$ & $19^{\circ} 41^{\prime} \frac{1}{2}$ & $\approx$ & $-5^{\prime}$ \\
\hline 18.03 .1504 & $20^{\circ} 3^{\prime}$ & $\approx$ & $20^{\circ} 40^{\prime} \frac{1}{2}$ & $\approx$ & $+7^{\prime} \frac{1}{2}$ \\
\hline 19.03 .1504 & $21^{\circ} 23^{\prime}$ & $\approx$ & $21^{\circ} 39^{\prime} \frac{2}{3}$ & $\approx$ & $+13^{\prime} \frac{2}{3}$ \\
\hline 27.03.1504 & $29^{\circ} 5 \mathrm{o}^{\prime}$ & $\approx$ & $29^{\circ} 51^{\prime}$ & $\approx$ & $+1^{\prime}$ \\
\hline
\end{tabular}

Kepler's goal in this calculation is to distribute the deviation, and indeed the four observations of Ptolemy turn out to be all in defect, while the Chaldean one is in excess. On the other hand, he remarks that taking out the Chaldean observation he can uniformly distribute the deviations among the four observation of Ptolemy within 9', but again he must change the mean motion and move the aphelion by about $2^{\circ}$. This represents a partial success that Kepler appears to be very proud of, since he says: "Conciliet eas propius, qui id potest, salva commensuratione orbium, per Tychonicas certissimas inventa" (reconcile them better, who can, provided the proportions among the orbits are respected, since we have firmly found them from Tycho).

But here the major troubles show up. Kepler realizes that he can find an appropriate secular equation for a given, not too long time 
interval, but he is unable to find one which is valid for all times. Coming indeed to recent observations (for him) so he writes. ${ }^{12}$

"At cum ex his epochis computarem postea Waltherianas et Regiomontani observationes exque iis appareret clarissime, $\hbar$ motus indigere æquatione seculari, eoque frustra nos medium affectare inter longe distantes, si inter se pugnent, nec in unam certis vicinis observationibus confirmatam commensurationem se cogi patiantur;

Nam quod Tychonicum attinet, videor ex oppositionibus acronychiis per totam triacontaëderis periodum jam sentiscere effectum æquationis secularis. Id autem fieri solet non in requatione maxima, tunc enim quantitas consistit, insensibili existente varietate, sed in rquatione prope nulla, tunc enim desinente adjectoria, incipiente subtratoria, vel e contrario, quantum potest maxima sentitur."

Thus, the first conclusion made by Kepler is that the secular equation can be calculated only relative to a short fixed period.

Let me add a remark. Kepler seems to assume that the planets possess a proper mean motion, and the secular equation consists in a periodic oscillation of the actual mean motion around the proper value that can be represented, e.g., by a sine function. What Kepler is attempting is to evaluate such a function (namely the mean velocity) using experimental data, but the lack of data does not allow him to complete his job.

Moreover he clearly misses all technical tools that have been developed more than half a century later, starting with the Methodus fluxionum et serierum infinitorum of Isaac Newton (1643-1727). His remark concerning Tycho's time is essentially that the derivative of a

12 "Moreover, using their epochs I have calculated the observations of Walther and Regiomontanus, and from them undoubtedly appears that the motion of Saturn is affected by a secular equation. Thus it is vain that we try to find an average between very distant observations, if they fight together and do not accept to be represented by a definite proportion confirmed by reliable and close observations; (...) Concerning Tycho's time indeed it seems to me that considering achronichous oppositions over a complete period of thirty years the effect of a secular equation faintly appears. This however usually does not happen for a maximal equation, since in that case the quantity remains almost constant due to an insensible change, but it rather happens close to a null value, when the quantities to be added before and to be subtracted after, or the contrary, reach the maximum attainable." 
Tab. 5. Observed positions of Mercury compared with the calculated ones.

\begin{tabular}{|c|c|c|c|c|c|}
\hline Date & \multicolumn{2}{|l|}{ Observed } & \multicolumn{2}{|l|}{ Calculated } & Difference \\
\hline 22.10 .1481 & $19^{\circ} 12^{\prime}$ & $\Omega$ & $19^{\circ} 3^{\prime}$ & $\Omega$ & $-9^{\prime}$ \\
\hline idem & $19^{\circ} 22^{\prime}$ & $\Omega$ & $19^{\circ} 3^{\prime}$ & $\Omega$ & $-19^{\prime}$ \\
\hline 3.11 .1481 & $4^{\circ} 36^{\prime}$ & $m$ & $5^{\circ} 2^{\prime}$ & $m$ & $+26^{\prime}$ \\
\hline idem & $4^{\circ} 4^{\prime}$ & $m$ & $5^{\circ} 2^{\prime}$ & $m$ & $+36^{\prime}$ \\
\hline 11.10 .1482 & $9^{0} 3^{2} \frac{1}{2}$ & $\Omega$ & $9^{\circ} 27^{\prime}$ & $\Omega$ & $-5^{\prime} \frac{1}{2}$ \\
\hline 16.01 .1488 & $23^{\circ} 40^{\prime}$ & $\approx$ & $23^{\circ} 40^{\prime}$ & $\approx$ & $\mathrm{o}^{\prime}$ \\
\hline 26.08 .1491 & $23^{\circ} 14^{\prime}$ & $\Omega$ & $23^{\circ} 13^{\prime}$ & $\delta$ & $-1^{\prime}$ \\
\hline 30.08 .1491 & $27^{\circ} 10^{\prime}$ & $\delta$ & $27^{\circ} 19^{\prime}$ & $\delta$ & $+9^{\prime}$ \\
\hline 31.08 .1491 & $28^{\circ} 34^{\prime}$ & $\Omega$ & $28^{\circ} 38^{\prime}$ & $\Omega$ & $+4^{\prime}$ \\
\hline 2.09 .1491 & $1^{\circ} 17^{\prime}$ & $m$ & $1^{\circ} 30^{\prime} \frac{1}{2}$ & mp & $+13^{\prime} \frac{1}{2}$ \\
\hline 3.09 .1491 & $3^{\circ} 9^{\prime}$ & $m$ & $3^{\circ} 3^{\prime}$ & $m$ & $-6^{\prime}$ \\
\hline 9.09 .1491 & $13^{\circ} 27^{\prime}$ & $m$ & $13^{\circ} 21^{\prime}$ & $m$ & $-6^{\prime}$ \\
\hline 9.01 .1504 & $3^{\circ} 36^{\prime} \frac{1}{2}$ & 6 & $3^{\circ} 3^{\prime}$ & 6 & $+1^{\prime} \frac{1}{2}$ \\
\hline 10.01 .1504 & $4^{\circ} 21^{\prime} \frac{1}{2}$ & 6 & $4^{\circ} 47^{\prime}$ & 6 & $+25^{\prime} \frac{1}{2}$ \\
\hline 11.03 .1504 & $17^{\circ} 34^{\prime} \frac{1}{2}$ & $\Upsilon$ & $17^{\circ} 39^{\prime}$ & $\Upsilon$ & $+4^{\prime} \frac{1}{2}$ \\
\hline 17.03 .1504 & $25^{\circ} 34^{\prime} \frac{1}{2}$ & $\Upsilon$ & $25^{\circ} 36^{\prime}$ & $\Upsilon$ & $+1^{\prime} \frac{1}{2}$ \\
\hline 18.03 .1504 & $26^{\circ} 24^{\prime} \frac{1}{2}$ & $\Upsilon$ & $26^{\circ} 36^{\prime}$ & $\Upsilon$ & $+1^{\prime} \frac{1}{2}$ \\
\hline 24.03 .1504 & $0^{\circ} 9^{\prime} \frac{1}{2}$ & $\gamma$ & $0^{\circ} 29^{\prime}$ & $\gamma$ & $+19^{\prime}$ \\
\hline idem & $0^{\circ} 29^{\prime} \frac{1}{2}$ & ૪ & $0^{\circ} 29^{\prime}$ & ૪ & $-\mathrm{o}^{\prime} \frac{1}{2}$ \\
\hline
\end{tabular}

function such as, e.g., the sine takes a maximum where its value is zero, while the function is almost constant in the vicinity of a maximum or minimum.

Now the problem is: where from does the proper mean motion come, and which is its value? The consideration concerning the observations of Tycho seem to suggest that Kepler's first idea was that the wanted value could coincide with the observed one at Tycho's time (actually, he later admits that this might be false). However, here comes the extra hypothesis that I have mentioned at the beginning of this section: Kepler strongly believes that at the beginning of time (the 


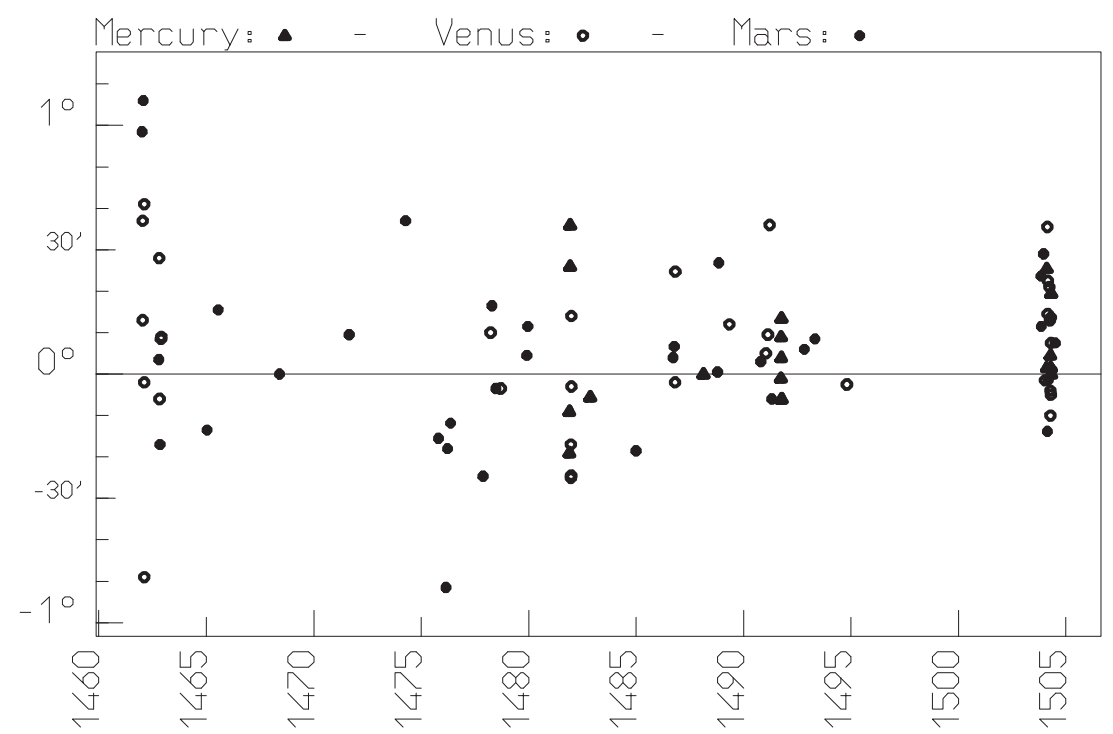

Fig. 2 - Difference between the longitudes of Mercury, Venus and Mars calculated by Kepler from the Tabulæ Rudolphinæ and the positions evaluated from the observations of Regiomontanus and Walther. In contrast with Fig. 1 no systematic deviation is observed here.

creation) the planets were in a privileged configuration, identified through the cardinal points of the orbit of the Earth. This is well stated in ch. XXIII of Mysterium Cosmographicum: ${ }^{13}$

"Certe non temere Deus instituit motus, sed ab uno quodam certo principio et illustri stellarum conjunctione, et in initio zodiaci, quod creator per inclinationem Telluris domicilii nostri effinxit, quia omnia propter bominem."

Kepler also attempted at determining the date of creation by combining calculations based on Genesis (which was very common at that time, typically giving dates around $4000 \mathrm{BC}$ ) and the configuration of the planets as given by the tables. In a note added to ch. XXIII of Mysterium Cosmographicum he claims that the Sun and the Moon

13 It is certain that God did not establish the motions inconsiderately, but from one well definite beginning and a privileged configuration of stars, and at the beginning of the zodiac, which has been moulded by means of the inclination of the Earth, our house, because everything has been created for the human beings." 
were created on July 24, 3993 BC. Fig. 3 fairly represents Kepler's thought. ${ }^{14}$ Besides the positions of the planets, he also tried hardly to show that at the same times also the aphelia and the nodes of the orbits were located at the same points, in a beautiful geometrical arrangement. This hypothesis actually represents for Kepler a major obstacle in trying to determine the secular equations. As it appears from the figure, some planets are not perfectly aligned with the cardinal points, what struggled Kepler a lot.

Kepler's note contains a long discussion on the old observations of Jupiter and Mars. In particular he shows that a mere change in the position of the aphelion will not explain the observed inequalities for Jupiter. Here he also raises several doubts concerning the correspondence among different calendars. Some of his doubts are dictated by his difficulty in fitting not just the data, but the initial configuration of the orbits. I skip this part, since for the purpose of justifying the need for secular equations it adds very little to the considerations on Saturn that I have reported. However, it is interesting to quote a sort of conclusion which fully enlights Kepler's claim, in the quotation at the beginning of this note, that many centuries of observations will pass before we can discover which secular equations we must introduce. Having remarked that perhaps an appropriate secular equation might fully remove the discrepancies with the observations of Ptolemy and Dionysius he says: $:^{15}$

14 Fig. 3 is found in a letter of Kepler to Peter Crüger (1580-1639) of February 18,1624 , where Kepler remarks that the calculated positions of the planets are very close to the cardinal points, and that trying to reduce them at the beginning of the zodiacal signs "ego nunc pene oculos ipsos computando perdo" (I'm almost consuming my eyes in computing). As to the calculation of the creation date, it may be curious to note that Jeremiah Horrocks (1618-1641) wrote in 1637 that compared to other similar calculations Kepler's one is "ingeniosa conjectura et non improbabilis" (an ingenious and non improbable conjecture).

15 "For he who will succeed in doing it will also convey information to posterity what must be added at Dionysius time, what at Ptolemy's time (since I do exactly that for the equinoxes at Ptolemy's time), and will also prescribe that they must annotate the excess or the defect for all future centuries (that certainly will be observed for all eccentrics). Then our descendants, on the basis of many data concerning excess and defect, will eventually succeed in studying a circular and ordered reconstruction and justifying it by calculations: and even, if needed, to change the velocity of the motions, so as to appropriately place our century in excess or in defect." 


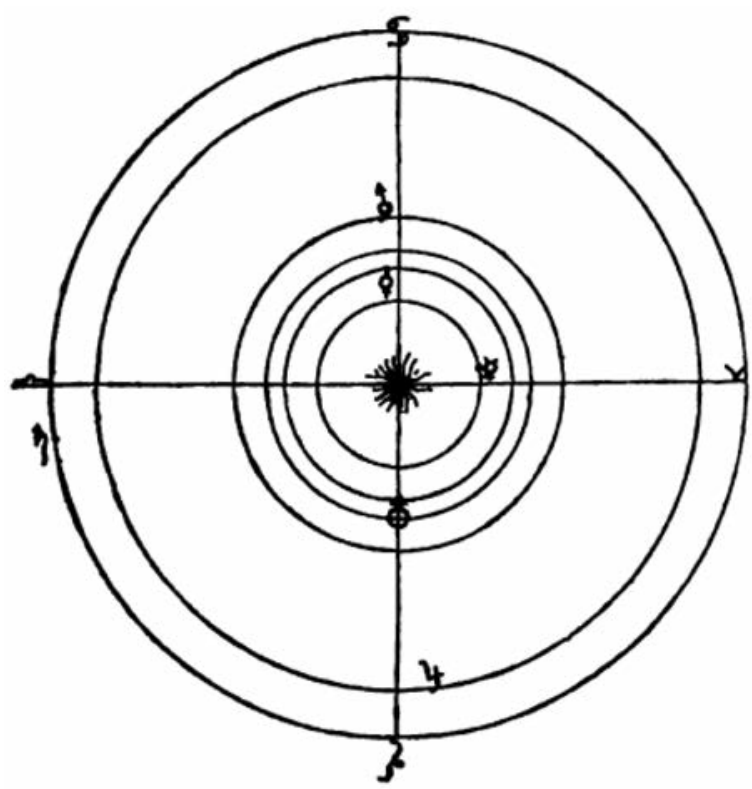

Fig. 3 - The configuration of the Solar System at creation time, according to one of the attempts by Kepler to place the planets at the cardinal points of the orbit of the Earth. The planets are close to the beginning of the constellations of $\uparrow$ (Aries), $\ni$ (Cancer), $\Omega$ (Libra) and 6 (Capricornus).

(Fig. taken from vol. VI of Johannis Kepleri Opera Omnia, pag. 30.)

"Nam qui boc fecerit, is admonitionem transmittere possit ad posteros, quid tempore Dionysii addendum, quid tempore Ptolemæi (sicut hoc ipsum ego facio in æquinotiis ad tempora Ptolemæi), jubens etiam ceterorum sequentium seculorum excessus vel defectus (qui quidem per totos eccentricos conspiciantur) annotare, tandemque eos, qui victuri sunt, ex pluribus idoneis defectibus et excessibus circularem et ordinatam restitutionem, qualis procul dubio est, investigare numerisque explicare: quin etiam, si aliter fieri nequeat, mutata motus celeritate, ipsum etiam boc nostrum seculum in excessu vel defectu collocare."

It seems evident here that the "secular equation" sought by Kepler is a periodic oscillation around an average value which reminds the epicycles of classical astronomy. His hope is that by accumulating enough experimental points one will be able to calculate a periodic function for the velocity. Furthermore, his conclusion fully agrees with the attitude of classical astronomers: the motion of the planets can be explained in terms 
of superposition of periodic motions, and the periods of such motions must be discovered through observations. As to the average values of the motions, they should coincide with the velocities at creation time. But if we remove the latter request and look only for the average observed values then we get very close to the attitude of astronomers after Lagrange and Laplace, as I will briefly illustrate in the next section. ${ }^{16}$

\section{After KePler}

A thorough discussion of the development of our knowledge after Kepler would not fit in a short note. Here I give abrief sketch. For more details see, e.g.,[20] or [23].

The note of Kepler, although unpublished, did not remain completely unknown: there are references in the literature to Kepler's conclusions, but usually in a generic form, typically saying that Kepler noticed remarkable inequalities in the motions of Saturn and Jupiter, and that Jupiter appears to accelerate, while Saturn seems to decelerate. Actually, this is what Kepler himself had communicated to his correspondents in some letters, without including further details. Furthermore, the significant increase of the number of observations together with the improvement of the precision confirmed more and more the existence of inequalities in the motion of the two biggest planets, somehow fulfilling Kepler's desire that observational data should be carefully collected.

The first attempt to include secular terms in tables is due to Edmond Halley (1656-1742), who in 1719 published new tables including secular corrections. However, he did not exploit Kepler's conjecture that one should look for a periodic behaviour (and likely he did not know it, since Kepler's note was still unpublished at that time). Pragmatically, he did just interpolate his data (around 1700) with

16 It may appear quite puzzling that Kepler does not explain how a varying mean motion may be reconciled with his hypothesis (which he strongly defends) that the proportions of the orbits and of the eccentricities are fixed. For, this seems to be in contrast with his third law, according to which one has $n^{2} a^{3}=$ const, where $n$ is the mean motion and $a$ the semimajor axis of the ellipse of a planet. Although Kepler in his note does not mention this problem, it seems to me that a straightforward interpretation is that the third law applies to the initial motion, which is assigned once for all to every planet and coincides with the average mean motion. 
Ptolemy's, and introduced a linear increment for the mean motion of Jupiter and a linear decrement for that of Saturn, that he called secular, as in Kepler. This, he claimed, could be enough in order to provide correct predictions for about 6000 years before and after 1700. Actually, a rough calculation based on his data and taking into account the third Kepler's law leads to the conclusion that about 2.7 millions of years ago Jupiter ad Saturn were on the same orbit, which is quite unlikely. But probably at Halley's time this was not a concern - and definitely it is not if one believes that the world was created less that 6000 years ago. What remains true is that Halley's secular corrections (linear in time for the mean motions) were taken as the paradigmatic reference for half a century and more, thus completely ignoring Kepler's idea that the secular motions should exhibit periods.

The accumulation of data showing with more and more evidence the inequalities of the two biggest planets raised the question whether the recent Newtonian theory of gravitation could be able to explain these phenomena. For it had been already pointed out by Newton that the mutual attraction among planets could induce a slow change in the orbits. The French Academy proposed three prizes, in 1748, 1750 and 1752, for solving this question. The 1748 and 1752 prizes were awarded to Leonhard Euler (1707-1783), while the 1750 one was not assigned. Euler's memoirs had the merit of creating the skeleton of perturbation theory, although, strictly speaking, his result was wrong: he calculated secular terms, in Halley's sense (i.e., linear in time for the mean motions), for the motion of Jupiter and Saturn which, however, had the same sign for both planets [1][2]. Thus, according to Euler's theory both planets should accelerate, in clear contrast with the observations. The error was partially corrected in 1762 by Joseph-Louis Lagrange (1736-1813): at least, he got the right sign, negative for Saturn and positive for Jupiter, still maintaining that the secular term was a linear one in the mean motion [12]. Eleven years later, in 1773, Pierre Simon de Laplace (17491827) remarked that by improving the approximation the secular terms found by Lagrange are canceled out by other contributions that Lagrange had neglected [16]. This question was eventually settled by Lagrange, who proved that no secular variation (linear in time) of the semimajor axes can occur in the approximation of first order in the masses of the planets, while a periodic variation may occur. Lagrange's result is remarkable, since the secular invariance of the semimajor axes of the planetary orbits represents a milestone in the research concerning 
the stability of the Solar System. Yet, his result cannot be considered as a conclusive one, since it holds true only in the first order approximation on the masses. On the other hand, at that time it had an unpleasant consequence: it implied that the "secular terms" introduced by Halley are not justified by Newton's theory; this meant that the problem of the inequalities of Jupiter and Saturn was reopened. The enigma was solved only in 1785, when Laplace discovered a long period perturbation due to the approximate resonance 2:5 between the periods of Jupiter and Saturn [17]: this perturbation has been named the great inequality. The period turns out to be about 900 years. Meanwhile Lagrange, soon followed by Laplace, had also developed a theory for the secular motions of nodes and perihelia together with the inclinations and the eccentricities of the orbits [13][14][15][16].

The theory developed by Lagrange and Laplace may be considered in some sense as a vindication of Kepler's intuition that the secular perturbations should be periodic. But it seems that nobody remarked it. On the other hand, two points deserve to be mentioned. The first one is that according to Lagrange and Laplace's theory all the orbital parameters are affected by periodic perturbations, including the semimajor axes and the eccentricities that Kepler had refused to change in view of the very precise observations of Tycho. The second point is that we had not to wait for many centuries of steady and careful observations, as predicted by Kepler. Both these points have a common explanation in the development of Newton's gravitational theory: the frequencies of the perturbation can be calculated on the basis of a mathematical model. Furthermore, the theory offers a method for determining at one time the most relevant deviations from the elliptic motions, because the equations tie all these quantities together. Indeed it is quite evident that discovering the exact form of a perturbation with a period of 900 years on the basis of less than two centuries of observations is a hard task, to say it fairly. Furthermore, separating the different contributions coming from slow changes in all parameters using only his own intuition is clearly impossible, and this had been indeed painful for Kepler.

Thus Kepler's intuition that the secular inequalities should be periodic seems to be confirmed by the theory. But, once again, reality is complex enough to escape our theories. A considerable amount of work has been spent during the XIX century in order to prove that the theory of Lagrange remains true at any order in the masses: the underlying idea is that the motion can be represented as a superposition of periodic com- 
ponents - the modern version of epicycles, that we rename Fourier series or quasi-periodic motion. On the other hand, the intuition that resonances could produce a destroying effect on the regularity of the orbits began to appear: this second aspect is connected with the hard problem of the so called small divisors (for an introductory reference see, e.g., [4]).

The representation of the planetary dynamic as composed of quasi-periodic motions has been seriously questioned at the end of the XIX century. On the one hand a brilliant Romanian student, Spiru Haretu, proved that secular terms (in Halley's sense) do appear in perturbation expansions at third order in the masses [3]. This result was soon forgotten, because new expansion methods were developed which at least in some cases could avoid such unwanted terms. On the other hand, the celebrated memoir of Poincare on the problem of three bodies revealed that a chaotic behaviour may show up. This seems to be the end of the game, but in 1954 Kolmogorov proved that quasi- periodic motions can persist, and they represent the majority of the possible motions if the masses of the planets are small enough [9]. But, what does "small enough" mean? This remains an open question [5]. It should also be mentioned that massive long term calculations of the planetary orbits have revealed that chaotic motions do indeed show up, although over time spans of several millions of years[18][19][21].

More than one century after Poincaré and more than fifty years after Kolmogorov we are still unable to give a complete answer to the problem of long term dynamics of the planets: the Solar System seems to interweave ordered and chaotic behaviour in an inextricable and intriguing manner. But this is a long story that can not fit in the present note. I conclude by emphasizing that the problem raised by Kepler is still there: we just succeeded in reformulating it in a definitely more refined but more complicated way.

\section{REFERENCES}

[1] L. Euler, Recherches sur la question des inégalités du mouvement de Saturne et de Jupiter, Pièce qui a remporté le prix de l'Académie Royale des Sciences, 1-123 (1748). Reprinted in: Opera Omnia, Ser. 2, Vol. 25, 45-157.

[2] L. Euler, Recherches sur les inegalités du mouvement de Jupiter et Saturne, Recueil des pièces qui ont remporté les prix de l'Académie Royale des Sciences 7, 1769, pp. 2-84. Reprinted in: Opera Omnia, Ser. 2, Vol. 26. 
[3] S.C. Haretu, Sur l'invariabilité des grands axes des orbites planétaires, Ann. Obs. Paris, Mémoires, 18, 1-39 (1885).

[4] A. Giorgilli, Small denominators and exponential stability: from Poincaré to the present time, Rend. Sem. Mat. Fis. Milano, LXVIII, 19-57 (1998).

[5] A. Giorgilli, U. Locatelli, Sulla stabilità del problema planetario dei tre corpi, Istituto Lombardo - Accademia di Scienze e Lettere, Rendiconti - Classe di Scienze, 143 (2009).

[6] Astronomia Nova, seu Physica Cœlestis tradita commentariis de motibus Stellæ Martis ex observationibus G.V. Tychonis Brahe; Jussu \& sumptibus Rudolphi II, Romanorum Imperatoris \& c. Plurium annorum pertinaci studio elaborata Pragæ, A.S.C.M.S. Mathematico Johanne Keplero, cum ejusdem C.M. privilegio speciali, Anno æræ Dionysianæ MDCIX. Reprinted in: Johannis Kepleri astronomi opera omnia, edidit Dr. Ch. Frisch, Frankfurti A.M. et Erlangæ Heyder \& Zimmer, MDCCCLX, Vol. III.

[7] J. Kepler, Consideratio observationum Regiomontani et Waltheri, in: Johannis Kepleri astronomi opera omnia, edidit Dr. Ch. Frisch Frankfurti A.M. et Erlangæ Heyder \& Zimmer, MDCCCLX, Vol. VI, pp. 725-774.

[8] J. Kepler, In tabulas Rudolphi præfatio, in: Johannis Kepleri astronomi opera omnia, edidit Dr. Ch. Frisch, Frankfurti A.M. et Erlangæ Heyder \& Zimmer, MDCCCLX, Vol. VI, pp. 666-674.

[9] A.N. Kolmogorov, Preservation of conditionally periodic movements with small change in the Hamilton function, Dokl. Akad. Nauk SSSR, 98, 527 (1954). English translation in: Los Alamos Scientific Laboratory translation LA-TR-7167; reprinted in: Lecture Notes in Physics 93.

[10] R.L. Kremer, Bernhard Walther's astronomical observations, Journal for the history of astronomy xi, 174-191 (1980).

[11] R.L. Kremer, The use of Bernhard Walther's astronomical observations: theory and observation in early modern astronomy, Journal for the history of astronomy xii, 124-132 (1981).

[12] J.L. Lagrange, Solution de différents problèmes de calcul intégral, Miscellanea Taurinensia, Tomo III (1762-1765). Reprinted in: Oeuvres de Lagrange, Gauthier-Villars, Paris (1870), tome I, p. 471-668.

[13] J.L. Lagrange, Recherche sur les équations séculaires des mouvements des noeuds et des inclinaisons des orbites des planètes, Mémoires de l'Académie Royale des Sciences de Paris (1774). Reprinted in: Oeuvres de Lagrange, Gauthier-Villars, Paris (1870), tome VI, p. 635-709.

[14] J.L. Lagrange, Théorie des variations séculaires des éléments des planètes. Première partie contenant les principes et les formules générales pour déterminer ces variations, Nouveaux mémoires de l'Académie des Sciences et Belles-Lettres de Berlin (1781). Reprinted in: Oeuvres de Lagrange, Gauthier-Villars, Paris (1870), tome V, p. 125-207.

[15] J.L. Lagrange, Théorie des variations séculaires des éléments des planètes. Seconde partie contenant la détermination de ces variations pour chacune des planètes principales, Nouveaux mémoires de l'Académie des Sciences et Belles- Lettres de 
Berlin(1782). Reprinted in: Oeuvres de Lagrange, Gauthier-Villars, Paris (1870), tome V, p. 211-489.

[16] P-S. de Laplace, Mémoire sur le principe de la gravitation universelle et sur les inégalités séculaires des planètes qui en dépendent, Mémoires de l'Académie Royale des Sciences de Paris (1773). Reprinted in: Oeuvres complètes de Laplace, Gauthier-Villars, Paris (1891), tome VIII, p. 201-275.

[17] P-S. de Laplace, Théorie de Jupiter et Saturne, Mémoires de l'Académie Royale des Sciences de Paris, année 1785, (1788). Reprinted in: Oeuvres complètes de Laplace, XI, p. 95.

[18] J. Laskar, A numerical experiment on the chaotic behaviour of the solar system, Nature, 338, 237-238(1989).

[19] J. Laskar, Large scale chaos in the solar system, Astron. Astroph. 287 (1994).

[20] J. Laskar, Lagrange et la Stabilité du Sytème Solaire, in: G. Sacchi Landriani e A. Giorgilli (eds): Sfogliando la Méchanique Analitique, LED edizioni, Milano (2008).

[21] L. Laskar, M. Gastineau, Existence of collisional trajectories of Mercury, Mars and Venus with the Earth, Nature 459, 817-819 (2009).

[22] J. Schöner (ed.), Scripta clarissimi mathematici M. Johannis Regiomontani, Nuremberg (1544). Republished in facsimile in: J. Regiomontanus: Opera collectanea, F. Schmeidler ed., 567-752, Osnabrück (1972). Walter's observations were also printed in: W. Snel: Coeli et siderum in eo errantium Hassiacæ, Leiden (1618) and in: L. Barettus [A.Curtz]: Historia coelestis, Augsburg (1666).

[23] C. Wilson, The great inequality of Jupiter and Saturn: from Kepler to Laplace, Archive for History of Exact Sciences 33, 15-290, (1985). 